$q i$ (energy) and shen (spirit). Qigong focuses on these three treasures to represent a holistic view of the human being. 'Eightsection brocades' is one of the many forms of health-promoting Chinese qigong.

A pilot study (Tsang et al, 2002) using a group of eight out-patients (two males and six females) suffering from chronic physical illnesses was conducted in Hong Kong. The mean age of the participants was 68 years (s.d.=10.7). Rating on the Geriatric Depression Scale showed that the participants had a certain degree of depressed mood, even though they did not carry a clinical diagnosis of depression. The participants received $1 \mathrm{~h}$ practice of qigong, twice a week, under the supervision of a qualified practitioner. As all participants had satisfactory standing balance, the standing-style eight-section brocades were used as the intervention protocol. The participants were asked to practise it daily (under the supervision of their relatives, who were also trained by the practitioner) for at least $30 \mathrm{~min}$ (in addition to the twice-weekly supervised practice in the hospital). The feedback from the participants showed that six of them $(75 \%)$ felt better in terms of their psychosocial functioning after the 12-week programme. Before 6 weeks of practice, only three $(37.5 \%)$, however, reported improvement. At an early stage, the feedback centred around physical function such as movement of the limbs and activities of daily living. At a later stage, the feedback then shifted more to psychological aspects. The improvement included feeling more relaxed, more comfortable, better sleep and being more optimistic. All of these reported improvements in psychosocial functioning are indicative of less depressed mood and improved quality of life. This preliminary report showed that qigong is promising as an alternative intervention for elderly people with depression and with chronic physical illness to improve their biopsychosocial health and possibly reduce their suicide rate. More systematic evaluation with larger samples and a longer period of intervention is now underway in Hong Kong.

Conwell, Y. (1996) Suicide in elderly patients. In Diagnosis and Treatment of Depression in Late Life (eds L. S. Schneider, C. F. Reynolds III, B. D. Lebowitz, et al). Washington, DC: American Psychiatric Press.
De Leo, D., Buono, M. D. \& Dwyer, J. (2002) Suicide among the elderly: the long-term impact of a telephone support and assessment intervention in northern Italy. British Journal of Psychiatry, 18I, 226-229.

Tsang, H. W. H., Cheung, L. \& Lak, D. (2002) Qigong as a psychosocial intervention for depressed elderly with chronic physical illnesses. International Journal of Geriatric Psychiatry, 17, II46-I154.

H.W. H. Tsang Department of Rehabilitation Sciences, The Hong Kong Polytechnic University, Hung Hom, Hong Kong

\section{Olanzapine toxicity in unconjugated hyperbilirubinaemia (Gilbert's syndrome)}

We have recently observed symptoms of toxicity caused by olanzapine at therapeutic dosages. Olanzapine metabolism was hampered because the patient had idiopathic unconjugated hyperbilirubinaemia (Gilbert's syndrome). As this is a frequent disorder in the general population (occurring in $10 \%$ of the European population), we feel that it is important to consider the possibility of Gilbert's syndrome before prescribing olanzapine.

A 19-year-old male with paranoid features and schizophrenic symptoms was treated with $2.5 \mathrm{mg}$ olanzapine for 2 days, which was increased to $5 \mathrm{mg}$ on the third day. On the fourth day, because of a suicide attempt and extreme agitation, the patient was admitted to a psychiatric centre. $\mathrm{He}$ was given oral doses of $10 \mathrm{mg}$ olanzapine and $5 \mathrm{mg}$ lorazepam. The patient was conscious on the sixth day but did not respond to verbal stimuli and his symptoms of mutism persisted over the next few days. Communication was possible by monosyllables on day eight. On day ten he was bradypsychic, oriented and capable of articulating short sentences with great effort. Speech returned to normal on day twelve. The patient described his experience as a sensation of not being able to find the words in his head. He had not previously displayed speech alterations, nor did they appear later.

Gilbert's syndrome and Crigler-Najjar syndromes type I and II are familial unconjugated hyperbilirubinaemias caused by genetic lesions involving a single complex locus encoding bilirubin uridine diphosphate - (UDP)-glucuronosyltransferase, which is involved in the detoxification of bilirubin by conjugation with glucuronic acid.

Over the past few years a number of different mutations affecting this gene have been characterised, in which a greater frequency of schizophrenia has been described (Miyaoka et al, 2000). Olanzapine is metabolised in the liver through direct glucuronidation reactions. Polymorphisms in glucuronosyltransferases, which often result in a decreased capacity for bilirubin glucuronidation, may have a significant impact on our capacity to detoxify and eliminate drugs and toxins (Mackenzie et al, 2000). Drug-mediated toxicity caused by genetic deficiency of UPD-glucuronosyltransferases is known (Burchell et al, 2000), as in the case of the administration of phenothiazine antipsychotics or tricyclic antidepressants. Mutism with olanzapine use has been reported in cases of overdose (Hanel et al, 1998; Cohen, 1999).

The use of therapeutic dosages of olanzapine can cause toxic symptoms if a lack of bilirubin UDP-glucuronosyltransferase is present. We should keep in mind idiopathic unconjugated hyperbilirubinaemia when prescribing olanzapine.

Burchell, B., Soars, M., Monaghan, G., et al (2000) Drug-mediated toxicity caused by genetic deficiency of UDP-glucuronosyltransferases. Toxicology Letters, I5 333-340.

Cohen, L. G., Fatalo, A., Thompson, B.T., et al (1999) Olanzapine overdose with serum concentrations. Annals of Emergency Medicine, 34, 275-278.

Hanel, E. A., Sandmann, M. C., Kranich, M., et al (1998) Neuroleptic malignant syndrome: a case report with recurrence with the use of olanzapine. Arquivos de Neuro-Psiquiatria, 56, 833-837.

Mackenzie, P., Miners, J. O. \& McKinnon, R. A. (2000) Polymorphisms in UDP glucuronosyltransferase genes: functional consequences and clinical relevance. Clinical Chemistry and Laboratory Medicine, 38, 889-892.

Miyaoka, T., Seno, H., Itoga, M., et al (2000) Schizophrenia-associated idiopathic unconjugated hyperbilirubinemia (Gilbert's syndrome). Journal of Clinical Psychiatry, 6I, 868-87I.

J. C. Martín-Escudero Department of Internal Medicine, Hospital Universitario Río Hortega, C/ Cardenal Torquemada S/N, Valladolid 470I0, Spain

A. Dueñas-Laíta, J. L. Pérez-Castrillón, V. Herreros-Fernández Department of Internal Medicine, Río Hortega University Hospital and University of Valladolid Medical School, Valladolid, Spain 